\title{
FRACTIONATION OF FULVIC ACIDS: CHARACTERISTICS AND COMPLEXATION WITH COPPER
}

\author{
Cheng-Fang Lin, ${ }^{a}$ Dar-Yuan Lee, ${ }^{b}$ Won-Tsai Chen ${ }^{a}$ \& Kevin S. Lo ${ }^{a}$ \\ ${ }^{a}$ Graduate Institute of Environmental Engineering, ${ }^{b}$ Department of Agricultural Chemistry, \\ National Taiwan University, Taipei, Taiwan, Republic of China
}

(Received 23 July 1993; accepted 11 January 1994)

\begin{abstract}
Two fulvic acid samples isolated from Suwannee river (International Humic Substance Society) and Feeitsuey reservoir were subjected to gel filtration chromatography (GFC) for molecular size fractionation. The GFC-eluted samples were separated into three groups corresponding to the molecular weight ranges: < 220, 220-1000, and 1000 4000. Fluorescence quenching techniques were employed for determining the conditional stability coefficient and kinetic parameters of copper complexation with the three fractions of fulvic acids. Experimental conditions were $p H 6,5 \times$ $10^{-5} \mathrm{M}$ total copper and $5 \mathrm{mg} C$ litre ${ }^{-1}$ of fulvic acids. The conditional stability coefficients of the fulvic acid fractions were in the order of $0.9-3.3 \times 10^{5} \mathrm{M}^{-1}$, and the forward and reverse rate constants were in the order of 6.9-12.4 $\times$ $10^{3} \mathrm{M}^{-1} \mathrm{~s}^{-1}$ and 3.5-8.0 $\times 10^{-2} \mathrm{~s}^{-1}$. Information could be useful in modelling copper transport in the hydrosphere.
\end{abstract}

\section{INTRODUCTION}

With increasing instances of water and/or soil contamination by heavy metals in Taiwan (Wang et al., 1989), the fate of heavy metals in soil and/or aquatic systems is of concern. Humic substances, a common component present in the soil and natural water bodies, appear to be the major metal-complexing ligands and, hence, play a significant role in the environmental fate, bioavailability, toxicity, and mobility of heavy metals in the biosphere (Allen et al., 1980; Dzombak et al., 1986; Weber, 1988; Oden et al., 1993). In recent years, several studies have been advanced to characterize the nature and properties of humic substances extracted from different soils and aqueous environment, to investigate the binding capacity for metals, and to determine the conditional stability constant of metal-humic/fulvic acid combinations from various sources (Stevenson, 1976; Saar \& Weber, 1980a; Ryan \& Weber, 1982; Frimmel \& Christman, 1988; Rate et al., 1993). The humic and fulvic acids previously isolated have been shown to contain many oxygen-containing groups. These functional groups are capable of binding with metal ions, and are thus responsible for metal complexation. The interactions between humic/fulvic acids and metal ions may be quantified by the binding capacity of organic ligands and the conditional stability constants of the reactions. Due to the complex nature of humic/fulvic acids, several models such as the single ligand, multiligand, discrete and continuous ligand models have been developed to describe the mass distribution of metals and ligands (Dzombak et al., 1986; Grimm et al., 1991; Nederlof et al., 1992; de Wit et al., 1993a,b). For example, Grimm et al. (1991) developed a continuous multiligand distribution model to predict the stability constant of $\mathrm{Cu}$ complexation with humic material, assuming normal distribution of the stability constants of specific binding sites. Considering the electrostatic effects, de Wit et al. (1993b) successfully applied the double layer theory to determine the intrinsic constant of proton binding to humic substances. Nederlof et al. (1992) compared various methods that were used to analyze complexation in the heterogeneous ligand systems and concluded that the new local isotherm approximation method better matched experimental results.

Unfortunately, these models, especially the discrete and continuous ligand models, are seldom verified with reference humic or fulvic acid samples. The difficulty is attributed to the identification of the 'binding site'; no single technology is available for this purpose. One way to elucidate the complex nature of humic/fulvic acids is to fractionate the mixed compounds and to study the thermodynamic and kinetic behavior of the separated compounds. Studies of fractionation and investigation of the polydiversity of the humic substances have been previously performed (Swift \& Posner, 1971; Levesque, 1972; Smith, 1976; Sposito et al., 1976). However, information about the thermodynamic and kinetic properties of fractionated humic/fulvic acids complexation with heavy metals is almost non-existent. In this work, gel filtration chromatography was used to fractionate two fulvic acids. The fractionated samples were then used for quantifying the complexing characteristics of fulvic acids with copper(II), using the fluorescence quenching method and a stopped-flow technique. In conjunction with pollutant transport models, the results should be useful for quantifying transport of the metal in the natural environment.

\section{MATERIALS AND METHODS}

Fulvic acid

The fulvic acids used in this work originated from two sources. Suwannee river reference fulvic acid (SRRFA) 
was obtained from the International Humic Substance Society (IHSS). The SRRFA is well characterized and its isolation method and chemical properties have been reported previously; the organic carbon content is approximately 53.5\% (Thurman \& Malcolm, 1981 1983). The second type of fulvic acid was isolated from sediment of Feeitsuey Reservoir, which supplies drinking water to northern Taiwan. The isolation methods followed the procedure of IHSS (IHSS, undated). Approximately, $1.6 \mathrm{~g}$ of powdered fulvic acid was obtained from $2.3 \mathrm{~kg}$ of airdried sediment. The fulvic acid extracted was hydrogensaturated and designated Feeitsuey Reservoir fulvic acid (FTFA). Solutions of SRRFA or FTFA were prepared by dissolving appropriate amounts of freeze-dried SRRFA or FTFA in water (Milli-Q reagent water) and filtered through a $0.45 \mu \mathrm{m}$ cellulose nitrate filter membrane. The filtrate was designated SRRFA or FTFA solution.

\section{Fractionation of fulvic acids by gel filtration chromatography}

The two types of fulvic acid solutions were thereafter subjected to gel filtration chromatography (GFC) for fractionating fulvic acid compounds (Levesque, 1972; Sposito et al., 1976). The fulvic acid solutions $\left(4 \mathrm{ml} ; 5 \mathrm{mg} \mathrm{ml}^{-1}\right.$, $\mathrm{pH}$ 6) together with the $\mathrm{NaClO}_{4}(0.02 \mathrm{M})$ eluant solution were introduced into the bed of a Pharmacia C26 column packed carefully with pre-swollen Sephadex G-50 fine gel. The total volume $(V$, of the gel was $450 \mathrm{ml}$, and the gel void volume $\left(V_{0}\right)$, determined by passing Dextran Blue (molecular weight $=2 \times 10^{6}$ ) through the column, was $200 \mathrm{ml}$. To minimize the effects of charge repulsion and adsorption, $0.02 \mathrm{M} \mathrm{NaClO}_{4}$ solution at $\mathrm{pH} 6$ was employed as eluant (Swift \& Posner, 1971). A peristaltic pump and Mariotte flask were used to maintain a constant hydraulic head and flow rate of $1 \mathrm{ml} \mathrm{min}$. The fractionated samples were collected in increments of $5 \mathrm{ml}$ using an automatic fraction collector.

Each $5 \mathrm{ml}$ of eluted fulvic acid was analyzed for absorbance at $254 \mathrm{~nm}$, total organic carbon (TOC), and fluorescence intensity. The organic carbon concentrations of SRRFA and FTFA solutions were determined with a TOC analyzer (OIC Model 700). The absorbance of fulvic acid samples was measured using a Jasco UV/VIS spectrophotometer. Spectrofluorometric measurements were accomplished with an Aminco SLM 8000 spectrofluorophotometer. The fluorescence excitation and emission wavelengths were set at 330 and 450 $\mathrm{nm}$, respectively, in the SRRFA solution and at 340 and $440 \mathrm{~nm}$ in the FTFA solution. These wavelengths were set based on the fluorescence excitation and emission maxima, and they were very similar to those found for fulvic acid reported by other researchers (Miano et al., 1988). Eluted fulvic acids were then grouped into three fraction sizes: elution volume from 200 to $300 \mathrm{ml},>300$ to $400 \mathrm{ml}$, and $>400$ to $500 \mathrm{ml}$. These three $100 \mathrm{ml}$ fractionated fulvic acids were used for equilibrium and kinetic experiments with $\mathrm{Cu}(\mathrm{II})$ complexation.

The elution parameter, $K_{a v}$, a relative measurement of molecular size (or molecular weight, $\mathrm{MW}$ ) was calculated using the equation $K_{a v}=\left(V_{e}-V_{o}\right) /\left(V_{t}-V_{o}\right)$, where $V_{e}$ is the elution volume for a given fraction and $V_{\text {, }}$ and $V_{o}$ are designated earlier (Skoog, 1985). Using reference materials with known MW (dextrose, PEG 400, PEG 1000, and PEG 4000), the relationship between $K_{a v}$ and MW was established as

$$
K_{a v}=2.084-0.536 \times \operatorname{logMW}
$$

$R^{2}$ (correlation coefficient) $=0.997$. The MW of any given fulvic acid fraction can therefore be calculated from the measured $V_{e}$ value.

\section{Equilibrium titration experiment}

Discontinuous titration of the fulvic acid or fractionated fulvic acid solutions with $\mathrm{Cu}\left(\mathrm{ClO}_{4}\right)_{2}$ solution was performed at $25^{\circ} \mathrm{C}$. Fulvic acid solutions $(100 \mathrm{ml})$ containing $0.01 \mathrm{M} \mathrm{NaClO}_{4}$ and $5 \mathrm{mg} \mathrm{C}$ litre ${ }^{-1}$ were placed into plastic beakers and small increments of $0.1 \mathrm{M}$ $\mathrm{Cu}\left(\mathrm{ClO}_{4}\right)_{2}$ solution were added carefully with a micropipette. After the desired total $\mathrm{Cu}$ concentration was achieved, the solutions were stirred by a magnetic stirrer and the $\mathrm{pH}$ was adjusted to $6 \cdot 0 \pm 0.1$ with $\mathrm{NaOH}$. After 5-10 min, about $2 \mathrm{ml}$ of the solution was taken for the fluorescence measurement. The equilibration interval of 5-10 min was established by preliminary kinetic studies. After measurement, the solutions were poured back into the plastic beaker for further addition of $\mathrm{Cu}(\mathrm{II})$. The titration series was carried out until light scattering was observed; the light scattering has been attributed primarily to the formation of flocculated particles of fulvic acid (Cabaniss, 1986).

\section{Kinetic experiments}

Kinetic analysis was performed with an Aminco SLM 8000 spectrofluorophotometer equipped with stoppedflow apparatus (Aminco FP-052). Solutions containing $10 \mathrm{mg} \mathrm{C}$ litre $^{-1}$ of SRRFA (or fractionated SRRFA), $10 \mathrm{mg} \mathrm{C}$ litre $^{-1}$ of FTFA (or fractionated FTFA), and $1 \times 10^{-4} \mathrm{M} \mathrm{Cu}$ (II) were used for the kinetic study. The fulvic acid and $\mathrm{Cu}$ solutions were placed into separate reagent reservoirs and pneumatically dispensed into a mixing chamber; hence, the concentrations of reactants in the mixing chamber were reduced by half. This kinetic setup is able to reach greater than $98 \%$ mixing efficiency at the observation point in less than $1.5 \mathrm{~ms}$; the minimum acquisition time and minimum acquisition rate (the data points recorded per unit time) are $10 \mathrm{~ms}$ and 1 point per ms, respectively. The fluorescence measurement was extended to $10-20 \mathrm{~s}$. In the kinetic runs, the $\mathrm{pH}$ value of reagents was preadjusted to $6.0 \pm$ 0.1 . It is believed that the small change in $\mathrm{pH}$ has no effect on the rate constant (Shuman, 1992).

\section{Data analysis}

The relationship between fluorescence emission intensities and metal complexation is based on the following assumption (Saar \& Weber, 1980b; Ryan \& Weber, 1982)

$$
I=\alpha_{M L} I_{M L}+\alpha_{L} I_{L}
$$


The total fluorescence intensity (I) is equal to the sum of the intensity of $\alpha_{M L} I_{M L}$ and $\alpha_{L} I_{L}$, where $\alpha_{M L}, I_{M L}, \alpha_{L}$ and $I_{L}$ are the molar ratio of metal-complexed organic ligand to total organic ligand, the limiting value of $I$ at maximum metal-ligand binding condition, the molar ratio of unbound organic ligand to the total organic ligand, and the fluorescence intensity of the initial free ligand in the absence of any added metal ions, respectively. Therefore, the fraction of organic ligand bound with metal ion can be expressed as

$$
\frac{[M L]}{\left[C_{L}\right]}=\frac{\left\{1-\left(I / I_{L}\right)\right\}}{\left\{1-\left(I / I_{L}\right)\right\}}
$$

where $[M L]$ is the molar concentration of metal complexed organic ligand and $\left[C_{L}\right]$ is the total molar concentration of organic ligand. For simplicity, it is assumed that a 1:1 stochiometric relationship exists between metal and organic ligand in the complexation reaction (Ryan \& Weber, 1982). Based on the mass balances on metal and organic ligand, the degree of complexation (eqn (3)) can be written in terms of the conditional stability constant $(K)$, total metal concentration $\left(C_{M}\right)$ and $C_{L}$

$$
\begin{aligned}
\frac{I_{L}-I}{I_{L}-I_{M L}}= & \frac{1}{2 K C_{L}}\left\{\left(K C_{L}+K C_{M}+1\right)\right. \\
& -\sqrt{\left(K C_{L}+K C_{M}+1\right)^{2}-4 K^{2} C_{L} C_{M}}
\end{aligned}
$$

Using the measured parameters $\left(I, I_{L}\right.$, and $C_{M}$ from metal titration experiments), the conditional stability constant, the total organic ligand concentration and the limiting fluorescence intensity at maximum metalligand binding condition $\left(I_{M L}\right)$ are obtainable by fitting $I$ and $I_{L}$ as a function of $C_{M}$ to eqn (4) using nonlinear regression techniques (Lee et al., 1992).

In the method of Saar and Weber $(1980 b)$, a single type of binding site was assumed in the derivation of equations. This may depict the apparent complexing capability of fulvic acids, but may not describe the heterogeneous nature of the system. Nevertheless, for simplicity, the fluorescence quenching method for determining the stability constant of the 'discrete ligands' was used in the present study. The conditional stability constant thus calculated was used to compute the reaction rate constants. As noted above, a $1: 1$ reaction between metal and organic ligand was presumed, i.e. $M+L=M L$. Although it is primitive, this assumption is widely used in most of the humic substances work.

Assuming an elementary and reversible reaction, the differential rate equation that applies to this complexation reaction is

$$
\frac{\mathrm{d}[M L]}{\mathrm{d} t}=k_{1}[M][L]-k_{-1}[M L]
$$

where $k_{1}$ and $k_{1}$ are the apparent forward and reverse rate constants. Replacing $k_{-1}$ with $k_{1} / K$, the concentrations of metal-ligand complex over time are computed from eqn (6) where $[M L]_{t},[M]_{t}$, and $[L]_{t}$ are the concentrations of metal-complexed organic ligand, free metal, and unbound organic ligand at time $t$ as the reaction proceeds.

$$
[M L]_{t+\Delta t}=[M L]_{t}+k_{1}\left([M]_{l}[L]_{t}-\frac{1}{K}[M L]_{t}\right) \Delta_{t}
$$

$[M L]_{t}$ is calculated from the observed fluorescence intensity such that $[M L]_{t}=[M L]_{e}\left(I_{L}-I_{t}\right) /\left(I_{L}-I_{e}\right)$, where $[M L]_{e}$ is the concentration of metal-complexed organic ligand at equilibrium and $I_{t}$ and $I_{e}$ are the fluorescence intensities of the reaction system at time $t$ and at equilibrium, respectively. With the given concentrations of metal and organic ligand and the observed values of $[M L]_{t}$ (eqn (6)), the apparent forward constant, $k_{l}$, was optimized.

\section{RESULTS AND DISCUSSION}

\section{Fractionation of fulvic acids}

The TOC, absorbance, and fluorescence of the fractionated fulvic acids as a function of molecular size are presented in Figs 1 and 2, respectively, for SRRFA and FTFA samples. From the elution diagrams, the MW of SRRFA ranges from 100 to 4000 , and that of FTFA is in the range of 100 to 8000 . At the position of maximum absorbance, the $\mathrm{MW}$ corresponds to approximately 800 for both SRRFA and FTFA. The
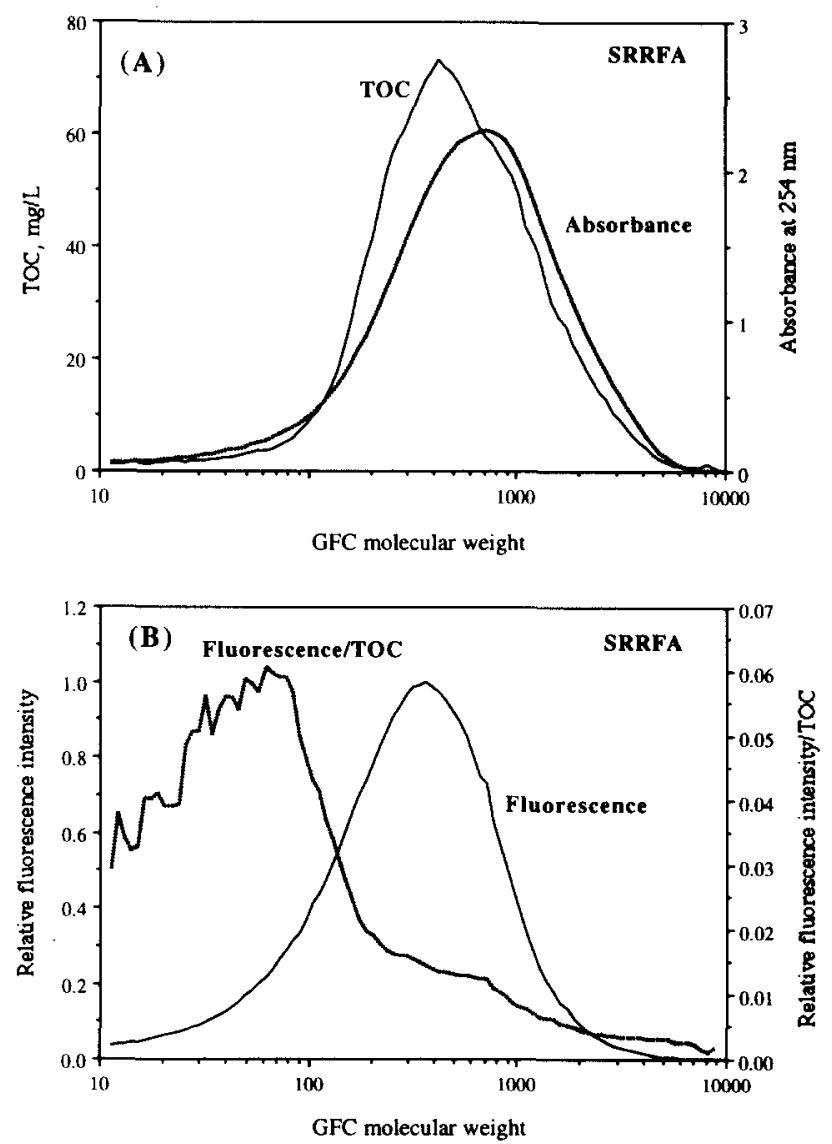

Fig. 1. Gel filtration chromatography (GFC) elution diagram of SRRFA as a function of molecular weight. (A) TOC and absorbance at $254 \mathrm{~nm}$. (B) Fluorescence intensity and fluorescence intensity/TOC. The GFC molecular weight in abscissa was converted from the elution volume using the elution parameter noted in the Materials and Methods section. 

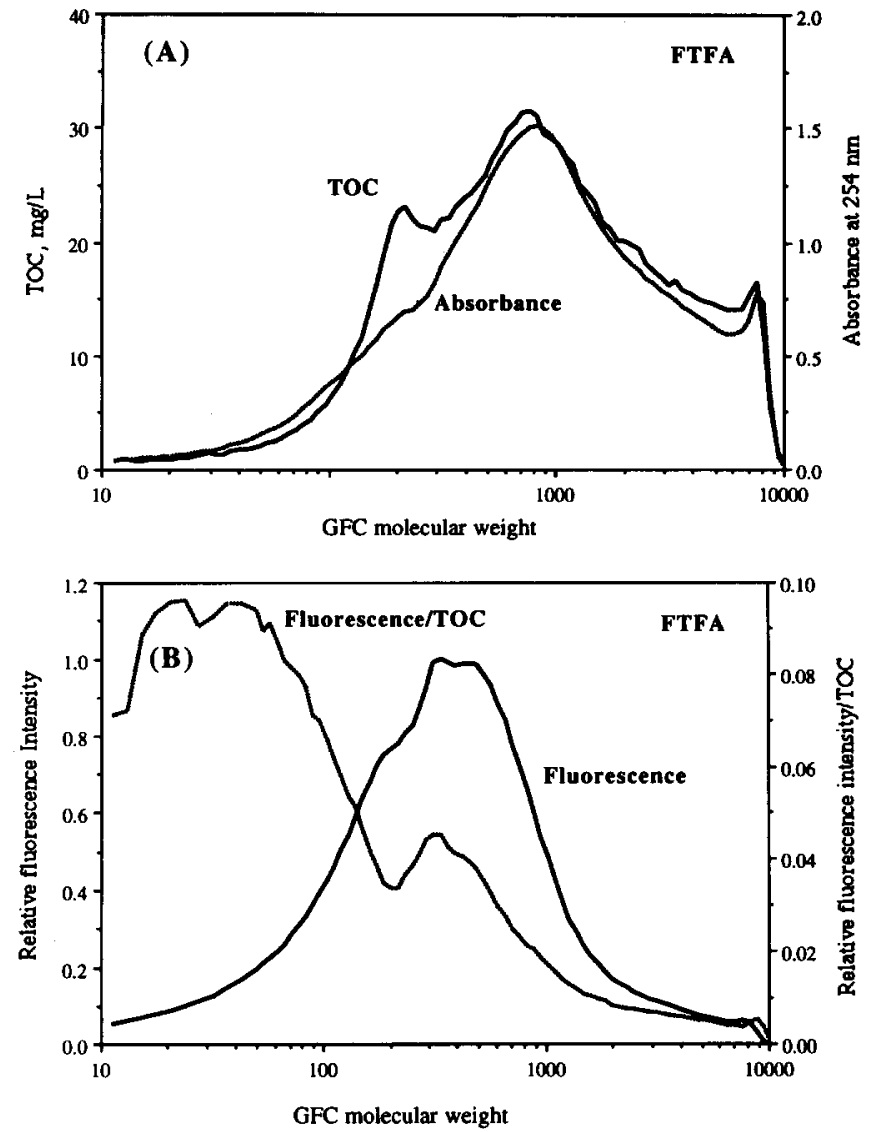

Fig. 2. Gel filtration chromatography (GFC) elution diagram of FTFA as a function of molecular weight. (A) TOC and absorbance at $254 \mathrm{~nm}$. (B) Fluorescence intensity and fluorescence intensity/TOC. The GFC molecular weight in abscissa was converted from the elution volume using the elution parameter noted in the Materials and Methods section.

calculated weight- and number-averaged molecuiar weights according to the plots of absorbance versus elution volume are 1674 and 855 , respectively, for SRRFA and 3993 and 1613 for FTFA. The numberaveraged MW results are quite consistent with those of others, e.g. 781 for the IHSS reference fulvic acid (tetrahydrofuran, THF) and 829 for the SRRFA (de Wit et al., 1993b), which confirms that the elution curves and the relationship between $K_{a v}$ and MW are acceptable. The fractionation of SRRFA and FTFA does not exhibit discrete molecular sizes; instead, the elution diagrams reflect that both the SRRFA and FTFA compounds have a continuous distribution of various complex species exhibiting different MWs. Also, polydispersity indices were 2.5 and 2.0 for FTFA and SRRFA, respectively. By comparison of the elution diagrams of both systems, FTFA seems to possess a less condensed molecular distribution and has more compounds with MW between 1000 and $8000 \mathrm{Da}$.

It appears that the variation in TOC as a function of molecular size is consistent with that of the absorbance for both SRRFA and FTFA systems. The variation of fluorescence, however, does not correlate well with that of absorbance and TOC. Nonetheless, the position where the peak fluorescence intensity occurs almost corresponds to the $V_{e}$ with the maximum TOC and
Table 1. Characteristies of the gel filtration chromatographyfractionated fulvic acids

\begin{tabular}{lcccc}
\hline $\begin{array}{c}\text { Fractionated } \\
\text { fulvic acid }\end{array}$ & $\begin{array}{c}\text { Elution } \\
\text { volume, ml }\end{array}$ & $\begin{array}{c}\text { Molecular } \\
\text { weight }^{a}\end{array}$ & $\begin{array}{c}\text { TOC, } \\
\text { mg C }_{\text {liter }}{ }^{-1}\end{array}$ & $\begin{array}{c}\text { Relative } \\
\text { fluorescence } \\
\text { intensity }^{b}\end{array}$ \\
\hline SRRFA-G1 & $200-300$ & $1000-4000$ & 24 & $0 \cdot 45$ \\
SRRFA-G2 & $300-400$ & $220-1000$ & 59 & $1 \cdot 10$ \\
SRRFA-G3 & $400-500$ & $<220$ & 16 & 1.69 \\
FTFA-G1 & $200-300$ & $1000-4000$ & 22 & 0.43 \\
FTFA-G2 & $300-400$ & $220-1000$ & 26 & $1 \cdot 62$ \\
FTFA-G3 & $400-500$ & $<220$ & 22 & 2.06
\end{tabular}

a The conversion of molecular weight from elution volume was noted in the text.

${ }^{b}$ The fluorescence intensity of the fractionated group relative to that of the unfractionated fulvic acid. The concentration of samples corresponds to $5 \mathrm{mg} \mathrm{C}$ litre ${ }^{-1}$.

absorbance. The fluorescence emitted per unit TOC for both systems is quite comparable qualitatively (Figs 1(B) and 2(B). The fluorescence emitted per unit TOC for low molecular size compounds ( $M W<100)$ is much higher than that of high molecular size compounds (MW > 1000). The observed results are consistent with those reported by Levesque (1972).

As noted in the preceding section, eluted fulvic acids were grouped into three fraction ranges: elution volume from 200 to $300 \mathrm{ml}, 300$ to $400 \mathrm{ml}$, and $>400$ to $500 \mathrm{ml}$. The relative nature of the fractionated fulvic acid groups in terms of elution volume, MW, TOC, and fluorescence intensity are summarized in Table 1. These six groups of fulvic acid compounds were subjected to equilibrium and kinetic experiments to determine the conditional stability constants and reaction rate constants of complexation with copper. As shown in Table 1 , the majority of organic carbon is present in group 2 which has a MW range of 220-1000. However, the fluorescent species are mostly present in the smaller molecular size fractions $(\mathrm{MW}<220)$. The results present in Table 1 confirm that fulvic acids extracted from naturally occurring dissolved organic matter are complex mixtures of different constituents. Different molecular size fractions may have different molecular structures and functional groups. Furthermore, the fulvic acid constituents are not evenly distributed over the entire molecular weight spectrum. Thus, fractionation of the fulvic materials and study of their thermodynamic and kinetic nature are of importance for understanding the extent of metal-fulvic acid complexation.

\section{Conditional stability coefficient of $\mathrm{Cu}$ complexation with fulvic acid fractions}

Based on the assumption that quenching of the fluorescence intensity of fulvic acid is proportional to the degree of metal-organic ligand complexation (Ryan \& Weber, 1982), a decrease in observed fluorescence intensity with increasing complexation with copper would be expected in the titration experiments. Figure 3 shows measured values of the relative fluorescence intensity $\left(I / I_{L}\right)$ as a function of the total $\mathrm{Cu}$ concentrations at $\mathrm{pH} 6$ for both FTFA and fractionated FTFA 


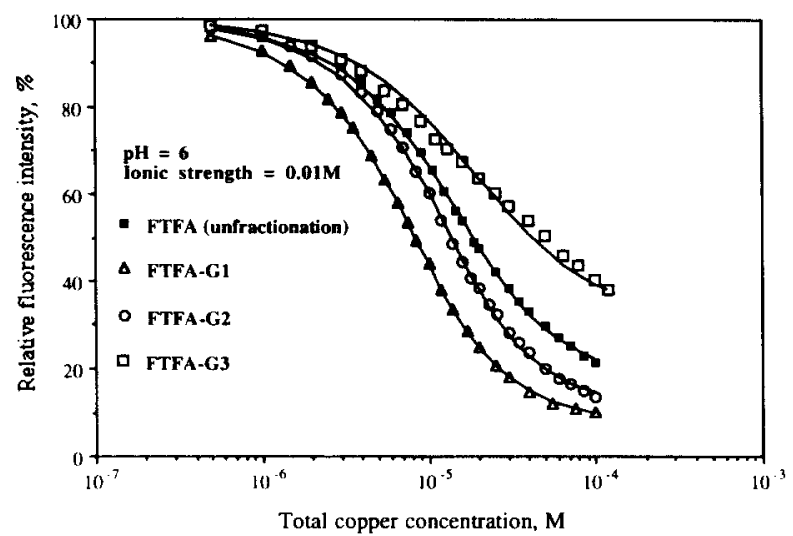

Fig. 3. Measured values of the relative fluorescence intensity as a function of the total $\mathrm{Cu}$ concentrations for FTFA systems. The concentrations of fulvic acids are $5 \mathrm{mg} \mathrm{C}$ litre $^{-1}$

systems. The values of the conditional stability constant, $K$, the residual fluorescence parameter, $I_{M L} / I_{L}$, and complexation capacity, $C_{L}$, were determined by applying eqn (4) with non-linear regression analysis. The optimized conditional stability coefficients for various fulvic acid fractions (SRRFA and FTFA) are listed in Table 2. The results indicate that the conditional stability coefficients of SRRFA and FTFA are $1.4 \times 10^{5}$ and $1.1 \times 10^{5} \mathrm{M}^{1}$, respectively, and those of the fractionated SRRFA or FTFA are in the order of $0.9 .-3.3 \times 10^{5} \mathrm{M}^{-1}$.

The results for conditional stability coefficients are comparable with those determined on the basis of fluorescence spectrometry for $\mathrm{Cu}$ (II) complexes with soil fulvic acid, surface-water organic matter, or leaf litter extract under similar $\mathrm{pH}$ and ionic strength conditions (Blaser \& Sposito, 1987). The determined apparent stability constants are also comparable to those obtained using other techniques, e.g. stability constants $(\log K)$ of $\mathrm{Cu}(\mathrm{II})$ complexation with humic material are 4.9 and 4.2 , by using the lanthanide ion probe and fluorescence quenching methods, respectively (Grimm et al., 1991). The complexing capacities and stability constants of fulvic acid with fluorescence quenching and ion-selective electrode methods have been compared (Saar \& Weber, 1980b; Ryan and Weber, 1982), and the results are quite consistent. As

Table 2. Conditional stability and kinetic parameters of the fractionated fulvic acids

\begin{tabular}{lccc}
$\begin{array}{l}\text { Fulvic } \\
\text { acid } \\
\text { samples }\end{array}$ & $\begin{array}{c}\text { Conditional } \\
\text { stability constant } \\
K\left(\mathrm{M}^{-1}\right)\end{array}$ & $\begin{array}{c}\text { Forward rate } \\
\text { constant } \\
k_{1}\left(\mathrm{M}^{-1} \mathrm{~s}^{-1}\right)\end{array}$ & $\begin{array}{c}\text { Reverse rate } \\
\text { constant } \\
k_{-1}\left(\mathrm{~s}^{-1}\right)\end{array}$ \\
\hline SRRFA & $1.4 \times 10^{5}$ & 5920 & 0.042 \\
SRRFA-G1 & $3.3 \times 10^{5}$ & 12390 & 0.037 \\
SRRFA-G2 & $2.2 \times 10^{5}$ & 10910 & 0.050 \\
SRRFA-G3 & $8.9 \times 10^{4}$ & 6880 & 0.077 \\
FTFA & $1.1 \times 10^{5}$ & 7540 & 0.069 \\
FTFA-G1 & $2.9 \times 10^{5}$ & 10250 & 0.035 \\
FTFA-G2 & $1.1 \times 10^{5}$ & 7340 & 0.067 \\
FTFA-G3 & $9.3 \times 10^{4}$ & 7450 & 0.080 \\
\hline
\end{tabular}

The experimental conditions were $\mathrm{pH} 6$, fulvic acid $5 \mathrm{mg} \mathrm{C}$ litre ${ }^{-1}$ total $\mathrm{Cu}$ (II) $5 \times 10^{-5} \mathrm{M}, 0.01 \mathrm{M} \mathrm{NaClO}_{4}$, and $25^{\circ} \mathrm{C}$. fulvic acids isolated from various sources exhibit different characteristics, comparison of the stability constants of various fulvic acids with different methods must be made with care.

It can be seen in Table 2 and from Fig. 3 that high molecular size fractions of fulvic acids will complex copper more strongly than lower molecular size fractions; this finding is in agreement with that of Sposito
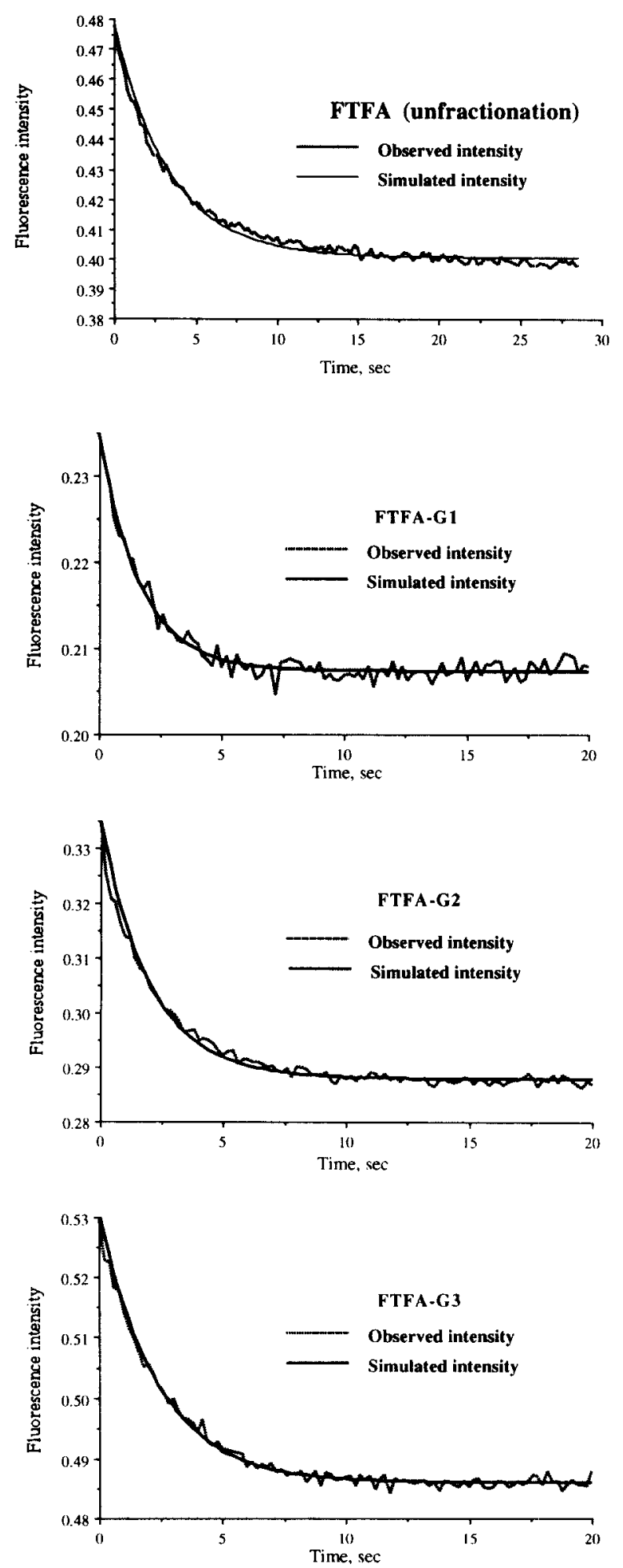

Fig. 4. Complexation kinetics at pH 6 in a system with $5 \times$ $10^{-5} \mathrm{M}$ copper and $5 \mathrm{mg} \mathrm{C}$ litre $\mathrm{e}^{-1}$ of FTFA and FTFA fractions. The observed intensity readings were simulated by the solid curve. 
et al. (1976). On the other hand, because the low molecular size fractions of dissolved organic matters may have more carboxyl binding sites, some investigators have reported that they will complex copper more strongly than higher molecular size fractions (Bender et al., 1970; Tan et al., 1970; Rashid, 1971; Smith, 1976).

\section{Rate coefficients of copper complexation with fulvic acid fractions}

The decrease in fluorescence intensity after mixing FTFA or the FTFA fractions and $\mathrm{Cu}$ (II) solution using the stopped-flow apparatus is illustrated in Fig. 4. Two kinetic runs for each fulvic acid and copper system were conducted and the experimental results indicated good reproducibility. The slight fluctuation of the fluorescence intensity observed is probably due to data collection and the integration process applied within a very short span $(0 \cdot 1-0 \cdot 3 \mathrm{~s})$. The original outputs from the spectrofluorophotometer are simulated by a curvefitting technique using nonlinear regression. Similar kinetic results of the SRRFA and $\mathrm{Cu}(\mathrm{II})$ reaction were obtained. Also from the kinetic diagrams, it is observed that the reaction of fulvic acids and copper complexation is fairly rapid and pseudo-equilibrium conditions can be achieved within 10 to $20 \mathrm{~s}$. The forward and reverse rate constants were calculated to be $7.5 \times 10^{3}$ $\mathrm{M}^{-1} \mathrm{~s}^{-1}$ and $6.9 \times 10^{-2} \mathrm{~s}^{-1}$, respectively, for the Cu-FTFA system, and $5.9 \times 10^{3} \mathrm{M}^{-1} \mathrm{~s}^{-1}$ and $4.2 \times 10^{-2} \mathrm{~s}^{-1}$ for the Cu-SRRFA system (Table 2). The results for the fractionated fulvic acids indicate that the forward rate constant is in the order of 5.9-12.4 $\times 10^{3} \mathrm{M}^{-1} \mathrm{~s}^{-1}$ and the reverse rate constant is in the order of 3.5-8.0 $\times$ $10^{-2} \mathrm{~s}^{-1}$. It is noted that high molecular size fractions have higher values of forward rate constants and lower values of reverse rate constants. As stated above, fulvic acids isolated from different sources may vary in nature and kinetic characteristics. The results have shown that the forward and/or reverse rate constants of SRRFA and FTFA fulvic acid fractions are of the same order of magnitude.

\section{CONCLUSION}

The two fulvic acids used in this study had GFC molecular weights in the range $100-4000$ for SRRFA and $100-8000$ for FTFA, respectively. The fluorescence intensity emitted from the fractionated fulvic acid compounds as a function of elution volume indicated that low molecular size fractions are the major fluorescent species. At the position of maximum absorbance, the molecular weight of the fractionated sample corresponded to 800 for both SRRFA and FTFA. The experiments performed in this study showed that the reaction of copper with fulvic acid is fairly rapid and pseudo-equilibrium conditions can be achieved within $10 \mathrm{~s}$. The results of GFC and complexation experiments also imply that the fulvic acids are complex mixtures containing multi-reacting components. Although the GFC-fractionated samples here illustrated that the high molecular size fractions of IHSS and FTFA seem to complex copper more strongly, the conditional stability constants as well as the reaction rate constants are about the same order of magnitude for the different fractions of SRRFA and FTFA. The conditional stability constant of fulvic acid- $\mathrm{Cu}^{2+}$ complexation is in the order of $10^{5} \mathrm{M}^{-1}$. The forward rate constant is in the order of 5.9-12.4 $\times 10^{3} \mathrm{M}^{-1} \mathrm{~s}^{-1}$ and the reverse rate constant is in the order of 3.5-8.0 $\times 10^{-2} \mathrm{~s}^{-1}$ for both IHSS and FTFA. These quantified thermodynamic and kinetic parameters are useful for modelling the fate and transport of copper in the aqueous environment, assuming the conditions are similar to those used in the present study.

\section{ACKNOWLEDGEMENTS}

Funding for this research was provided by the National Science Council of the Republic of China under grant No. NSC-82-0410-E-002-136. The authors specially acknowledge Dr W. Y. Lin, Dept. of Chemistry, National Taiwan University, for his assistance and discussion.

\section{REFERENCES}

Allen, H. E., Hall, R. H. \& Brisbin, T. D. (1980). Metal speciation: Effects on aquatic toxicity. Environ. Sci. Technol. , 4, 441-3.

Bender, M. E., Matson, W. R. \& Jordan, R. A. (1970). On the significance of metal complexing agents in secondary sewage effluents. Environ. Sci. Technol., 4, 520-1.

Blaser, R. A. \& Sposito, G. (1987). Spectrofluorometric investigation of trace metal complexation by an aqueous chestnut leaf extract. Soil Sci. Soc. Am. J., 51, 612-19.

Cabaniss, S. E. (1986). A potentiometric and fluorometric study of copper complexation by dissolved organic matter. $\mathrm{PhD}$, thesis, University of North Carolina, Chapel Hill, NC, USA.

Dzombak, D. A., Fish, W. \& Morel, M. M. (1986). MetalHumate interaction. 1. Discrete ligand and continuous distribution models. Environ. Sci. Technol., 20, 669-75.

Frimmel, F. H. \& Christman, R. F. (1988). Polymerization of humic substances in natural environments. In Humic substances and their role in the environment. John Wiley, New York, NY, pp. 45-58.

Grimm, D. M., Azarraga, L. V., Carreira, L. A. \& Susetyo, W. (1991). Continuous multiligand distribution model used to predict the stability constant of $\mathrm{Cu}$ (II) metal complexation with humic material from fluorescence quenching data. Environ. Sci. Technol., 25, 1427-31.

IHSS (undated). Extraction for IHSS references samples outline of procedure. International Humic Substances Society.

Lee, D. Y., Lin, C. F., Houng, L. M. \& Lo, K. S. (1992). Spectrofluorometric investigation of $\mathrm{Cu}$ and $\mathrm{Pb}$ complexation by dissolved organic matter. Chinese Inst. of Environ. Engr., 2, 51-6.

Levesque, M. (1972). Fluorescence and gel filtration of humic compounds. Soil Science, 113, 346-53.

Miano, T. M., Sposito, G. \& Martin, J. P. (1988). Fluorescence spectroscopy of humic substance. Soil Sci. Soc. Am. $J .$, 52, 1016-19.

Nederlof, M. M., Riemsdijk, W. H. \& Koopal, L. K. (1992). Comparison of semianalytical methods to analyze complexation with heterogeneous ligands. Environ. Sci. Technol., 26, 763-71.

Oden, W. I., Amy, G. L. \& Conklin, M. (1993). Subsurface interactions of humic substances with $\mathrm{Cu}$ (II) in saturated media. Environ. Sci. Technol., 27, 1045-51. 
Rashid, M. A. (1971). Role of humic acids of marine origin and their different molecular weight fractions in complexing di- and tri-valent metals. Soil Sci., 111, 298-306.

Rate, A. W., McLaren, R. G. \& Swift, R. S. (1993). Response of copper(II)-humic acid dissociation kinetics to factors influencing complex stability and macromolecular conformation. Environ. Sci. Technol., 27, 1408-14.

Ryan, D. K. \& Weber, J. H. (1982). Fluorescence quenching titration for determination of complexing capacities and stability constants of fulvic acid. Anal. Chem., 54, 986-90.

Saar, R. A. \& Weber, J. H. (1980a). Lead(II)-fulvic acid complexes conditional stability constants, solubility, and implication for lead(II) mobility. Environ. Sci. Technol., 14, 877-80.

Saar, R. A. \& Weber, J. H. (1980b). Comp:rison of spectrofluorometry and ion-selective electrode potentiometry for determination of complexes between fulvic acid and heavy-metal ions. Anal. Chem., 52, 2093-100.

Shuman, M. S. (1992). Dissociation pathways and species distribution of aluminum bound to an aquatic fulvic acid. Environ. Sci. Technol., 26, 593-8.

Skoog, D. A. (1985). Principles of Instrumental Analysis. Saunders College Publishing.

Smith, R. G. (1976). Evaluation of combined applications of ultrafiltration and complexation capacity techniques to nature waters. Anal. Chem., 48, 74-6.

Sposito, G., Holtzclaw, K. M \& Baham, J. (1976). Analytical properties of the soluble, metal-complexing fractions in sludge-soil mixture: II. Comparative structural chemistry of fulvic acid. Soil Sci. Soc. Am. J., 40, 691-7.

Stevenson, F. J. (1976). Stability constants of $\mathrm{Cu}, \mathrm{Pb}$ and $\mathrm{Cd}$ complexes with humic acids. Soil Sci. Soc. Am. J., 40, 665-72.

Swift, R. S. \& Posner, A. M. (1971). Gel chromatography of humic acid. Soil Sci. Soc. Am. J., 23, 237-49.

Tan, K. H., King, L. D. \& Morris, H. D. (1970). Complex reactions of zinc with organic matter extracted from sewage sludge. Soil Sci. Soc. Am. Proc., 35, 748-51.

Thurman, E. M. \& Malcolm, R. L. (1981). Preparative isolation of aquatic humic substances. Environ. Sci. Technol., $15,463-6$.

Thurman, E. M. \& Malcolm R. L. (1983). Structural study of humic substances: New approaches and methods. In Aquatic and Terrestrial Humic Materials, ed. R. F. Christman \& E. T. Gjessing. Ann Arbor Science, Ann Arbor, MI.

Wang, Y. P., Li, G. C. \& Chen, Z. S. (1989). Data base and distribution of heavy metals in Taiwan agricultural soil. Proc. 1st Workshop of Soil Pollution Prevention, Taiwan.

Weber, J. H. (1988). Binding and transport of metals by humic materials. In Humic Substances and Their Role in the Environment, ed. F. H. Frimmel \& R. F. Christman. John Wiley, New York, NY, pp. 165-78.

de Wit, J. C. M., van Riemsdijk, W. H. \& Koopal, L. K. (1993a). Proton binding to humic substances. 1. Electrostatic effects. Environ. Sci. Technol., 27, 2005-14.

de Wit, J. C. M., van Riemsdijk, W. H. \& Koopal, L. K. $(1993 b)$. Proton binding to humic substances. 2. Chemical heterogeneity and adsorption models. Environ. Sci. Technol., 27, 2015-22. 